\title{
Magnetic Structure and Dielectric State in the Multiferroic $\mathrm{Ca}_{2} \mathrm{CoSi}_{2} \mathrm{O}_{7}$
}

\author{
Minoru Soda $^{1}$, Shohei Hayashida ${ }^{1}$, Toshiya Yoshida ${ }^{1}$, Mitsuru Akaki², \\ Masayuki Hagiwara ${ }^{2}$, Maxim Avdeev ${ }^{3}$, Oksana Zaharko ${ }^{4}$, and Takatsugu Masuda ${ }^{1}$ \\ ${ }^{1}$ Neutron Science Laboratory, Institute for Solid State Physics, University of Tokyo, Tokai, Ibaraki 319-1106, Japan \\ ${ }^{2}$ Center for Advanced High Magnetic Field Science (AHMF), Graduate School of Science, Osaka University, \\ Machikaneyama 1-1, Toyanaka, Osaka 560-0043, Japan \\ ${ }^{3}$ Bragg Institute, Australian Nuclear Science and Technology Organization, Lucas Heights, New South Wales 2234, \\ Australia \\ ${ }^{4}$ Laboratory for Neutron Scattering and Imaging, Paul Scherrer Institut, CH-5232 Villigen PSI, Switzerland
}

\begin{abstract}
Magnetic structure of the multiferroic $\mathrm{Ca}_{2} \mathrm{CoSi}_{2} \mathrm{O}_{7}$ was determined by neutron diffraction techniques. Combination of the polycrystalline and single-crystal samples experiments revealed a collinear antiferromagnetic structure with the easy axis along $<100>$ directions. The dielectric state was discussed in the framework of the spin-dependent $d$ - $p$ hybridization mechanism, leading to the realization of the antiferroelectric structure. The origin of the magnetic anisotropy was discussed in comparison with the isostructural $\mathrm{Ba}_{2} \mathrm{CoGe}_{2} \mathrm{O}_{7}$.
\end{abstract}

\section{Introduction}

The relationship between magnetism and dielectricity has attracted great interest in the field of condensed matter physics ${ }^{1-4)}$ Among them multiferroics, in which the ferroelectricity is accompanied by the magnetic ordering, have been studied both experimentally ${ }^{5-7)}$ and theoretically. ${ }^{8-11)}$ The microscopic mechanisms are explained by the spinorbit coupling, which revealed the analytical relation between the spin structure and local electric polarization. For example, the multiferroic property in materials having cycloidal structures is explained by spin current mechanism, , 12-14) and that having a proper-screw structure is explained by the spin-dependent $d$ - $p$ hybridization mechanism. ${ }^{10,11,15,16)}$ The change of the magnetic structure with temperature or magnetic field affects the ferroelectricity on the basis of the analytical relation. ${ }^{17-19)}$

Recently, several multiferroic materials with a collinear structure have been studied. ${ }^{20-24)}$ One example is a squarelattice antiferromagnet $\mathrm{Ba}_{2} \mathrm{CoGe}_{2} \mathrm{O}_{7}$, the multiferroic properties of which have been explained by the $d-p$ hybridization mechanism. ${ }^{23)}$ A large single-ion anisotropy $D$ of easy-plane type and a small spin-nematic interaction $J_{p}$, which is responsible for in-plane anisotropy, play an important role in the material. ${ }^{25,26)}$ As for the spin dynamics, the large $D$ term induces an optical flat mode, which corresponds to an electric-field active mode confirmed by electromagnetic wave absorption experiments. ${ }^{27)}$ The spin-nematic interaction is equivalent to the interaction between local electric polarizations, and the dielectric energy was probed in low energy range by an inelastic neutron scattering (INS) ${ }^{25)}$ Furthermore, the direction of the magnetic moment in the $c$-plane was controlled by the electric field, which is quantitatively explained by the Hamiltonian including the dielectric energy estimated from the INS experiment. ${ }^{26)}$

Here, we focus on an isostructural material $\mathrm{Ca}_{2} \mathrm{CoSi}_{2} \mathrm{O}_{7}$ in order to study physical properties of the material having a different set of $D, J_{p}$, and additional orthorhombic anisotropy $E$. The $\mathrm{Ca}_{2} \mathrm{CoSi}_{2} \mathrm{O}_{7}$ has a two-dimensional network of $\mathrm{CoO}_{4}$ and $\mathrm{SiO}_{4}$ tetrahedra as shown in Fig. 1(a). $\mathrm{Co}^{2+}$ ions have spin
$S=3 / 2$ and form a square lattice. Below $T_{\mathrm{N}}=6 \mathrm{~K}$, a magnetic order appears, and the electric polarization is induced by the magnetic field $H \cdot{ }^{28,29)}$

In the present study, neutron diffraction measurements have been performed to clarify the magnetic structure of $\mathrm{Ca}_{2} \mathrm{CoSi}_{2} \mathrm{O}_{7}$. The determined structure was a collinear antiferromagnetic one with the easy axis along $<100>$ directions. Consideration on the multiferroic property in the framework of the spin-dependent $d$ - $p$ hybridization mechanism led to the same dielectric state as that of the isostructural $\mathrm{Ba}_{2} \mathrm{CoGe}_{2} \mathrm{O}_{7}$. The discussion on the magnetization data on the basis of the determined magnetic structure revealed that the origin of the in-plane anisotropy that dominates the multiferroic property is orthorhombic anisotropy $E$.

\section{Experimental Details}

The single crystals of $\mathrm{Ca}_{2} \mathrm{CoSi}_{2} \mathrm{O}_{7}$ were grown by the floating-zone method. ${ }^{28)}$ The polycrystalline sample was obtained by pulverizing the single crystal. The magnetiza-
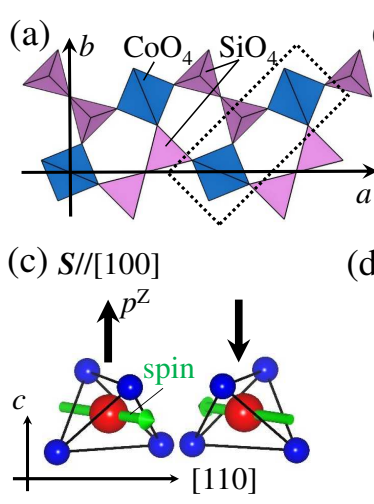

(b)

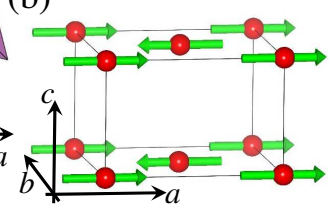

(d) $S / /[110]$

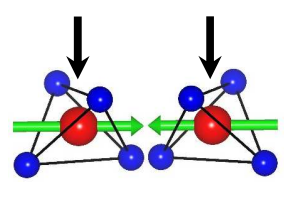

Fig. 1. (color online) (a) Two-dimensional network of $\mathrm{CoO}_{4}$ and $\mathrm{SiO}_{4}$ tetrahedra in $\mathrm{Ca}_{2} \mathrm{CoSi}_{2} \mathrm{O}_{7}$. (b) Schematic magnetic structure of $\mathrm{Ca}_{2} \mathrm{CoSi}_{2} \mathrm{O}_{7}$. (c,d) Dielectric state of $\mathrm{CoO}_{4}$ tetrahedron for the magnetic structures with the easy axes along $(c)<100>$ and $(\mathrm{d})<110>$. Green arrows indicate the $\mathrm{Co}^{2+}$ spin $(S)$, and black arrows indicate the local electric polarization along the $c$-axis $\left(p^{Z}\right)$. 


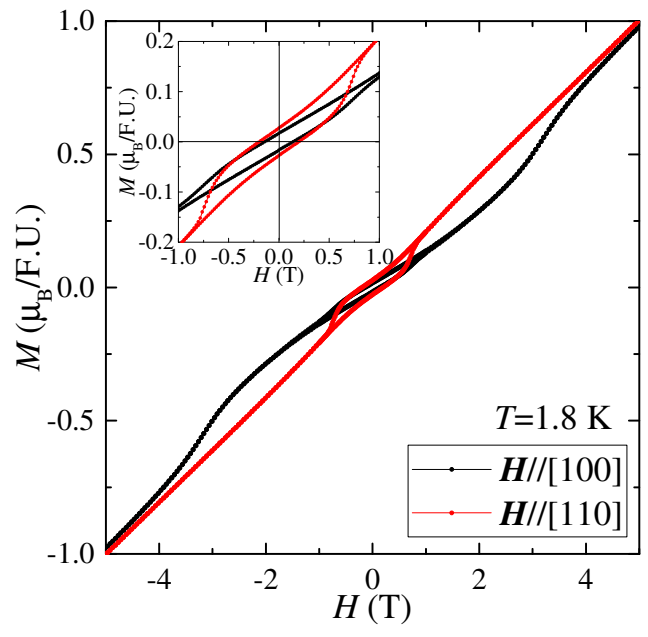

Fig. 2. (color online) Magnetic-field dependence of magnetization on single crystal measured at $T=1.8 \mathrm{~K}$ for $\boldsymbol{H} \|[100]$ and $\boldsymbol{H} \|[110]$. Inset shows the low-field region of the magnetization.

tion was measured by a commercial SQUID magnetometer. Powder neutron diffraction experiments were performed at ECHIDNA spectrometer installed at OPAL in ANSTO, Australia. The used neutron wavelength with Ge (331) monochromator was $2.4395 \AA$. The single-crystal neutron diffraction experiment was carried out using TriCS spectrometer installed at SINQ in PSI, Switzerland. The used neutron wavelength with Ge (311) monochromator was $1.18 \AA$. The scattering plane was the $a-b$ plane, and throughout this paper, we use the tetragonal unit cell with the space group $P \overline{4} 2_{1} m$ where the lattice constants are $a=b=7.844 \AA$ and $c=5.027 \AA$. The sample was cooled by using a liquid-helium cryostat.

\section{Results and Analysis}

\subsection{Magnetization}

Figure 2 shows the magnetic field $(H)$ dependence of the magnetization $(M)$ on single-crystal measured at $1.8 \mathrm{~K}$ for $\boldsymbol{H} \|[100]$ and $\boldsymbol{H} \|[110]$. Hysteresis induced by the weak ferromagnetic component is observed below $H=1 \mathrm{~T}$. The magnetization for $\boldsymbol{H} \|[100]$ shows nonlinear behaviour at about 3.5 $\mathrm{T}$, while the magnetization for $\boldsymbol{H} \|[110]$ indicates almost linear behaviour at $1 \mathrm{~T}<H<5 \mathrm{~T}$. These are in contrast with the isostructural material $\mathrm{Ba}_{2} \mathrm{CoGe}_{2} \mathrm{O}_{7}$; the $M$ in the field $\boldsymbol{H} \|[110]$ is nonlinear and the $M$ in the field $\boldsymbol{H} \|[100]$ is linear .$^{25)}$ The inset of Fig. 2 depicts the low field region of magnetization. This is consistent with the previous study of the magnetization. ${ }^{29)}$

\subsection{Powder Neutron Diffraction}

The main panel in Fig. 3(a) shows the neutron powderdiffraction patterns measured at $3.5 \mathrm{~K}$ and $20 \mathrm{~K}$. The inset shows the calculation of nuclear reflections on the basis of the averaged crystal structure with the space group $P \overline{4} 2_{1} m \cdot{ }^{30)}$ Figure 3(b) shows the magnetic intensities obtained by subtracting the data at $20 \mathrm{~K}$ from that at $3.5 \mathrm{~K}$. Several magnetic reflections are observed in the low angle region, and all the magnetic peaks are indexed by a propagation vector $(0,0,0)$. The obtained vector coincides with that in $\mathrm{Ba}_{2} \mathrm{CoGe}_{2} \mathrm{O}_{7} \cdot{ }^{31)}$ In Fig.
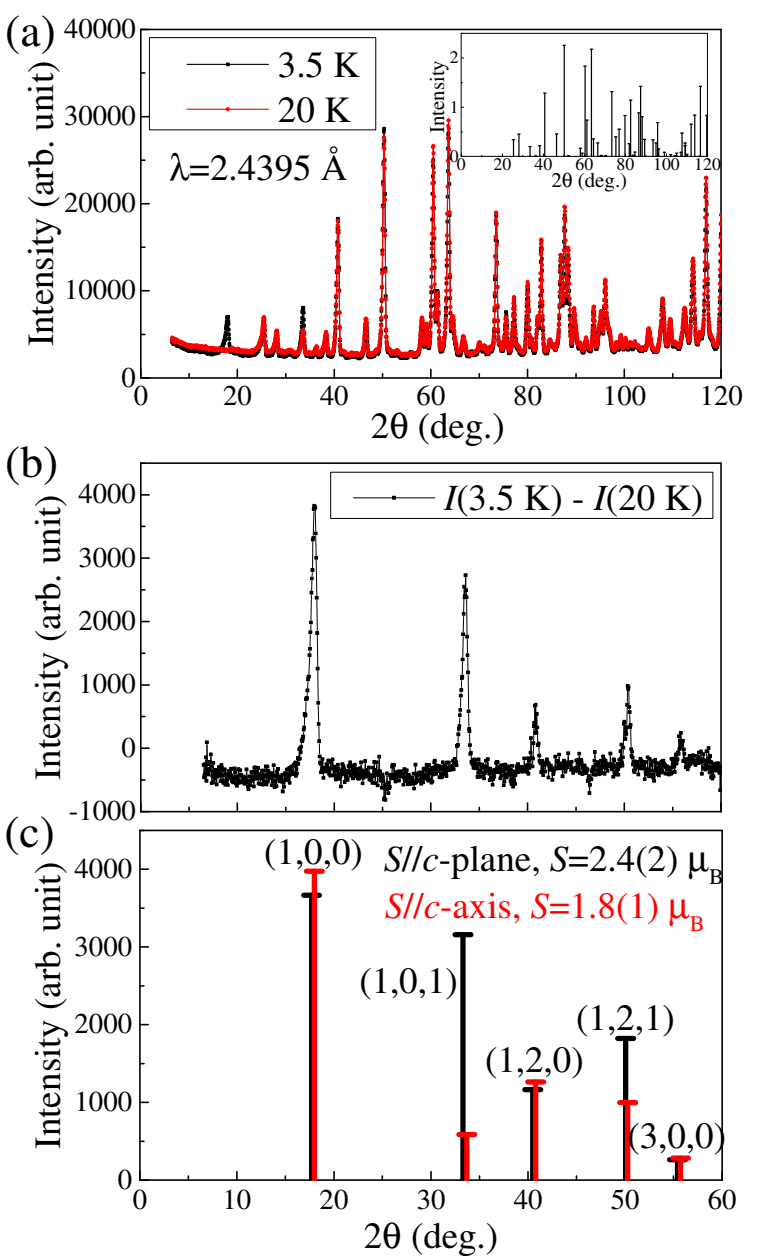

Fig. 3. (color online) (a) Neutron-powder-diffraction patterns measured at $3.5 \mathrm{~K}$ and $20 \mathrm{~K}$. Inset shows the calculated intensities of nuclear reflections with the space group $P \overline{4} 2_{1} m$. (b) Magnetic intensities obtained by subtracting the data at $20 \mathrm{~K}$ from that at $3.5 \mathrm{~K}$. (c) Magnetic intensities calculated for the collinear-magnetic structures with the easy axis in the $c$-plane and along the $c$-axis.

3(c), the magnetic intensities are calculated for two models; the collinear antiferromagnetic structure with the spins confined in the $c$-plane and that with the spins directed along the $c$-axis. It is noted that the direction of the magnetic moment in the $c$-plane can not be determined by a powder neutrondiffraction technique because the technique is not sensitive to magnetic domains. In the calculation, the isotropic magnetic form factor of $\mathrm{Co}^{3+}$ was used. ${ }^{32)}$ The estimated magnitude of the Co-magnetic moment in the former model is 2.4(2) $\mu_{\mathrm{B}}$ and that in the latter is $1.8(1) \mu_{\mathrm{B}}$. The former model reproduces the experiment better, and we found that $\mathrm{Ca}_{2} \mathrm{CoSi}_{2} \mathrm{O}_{7}$ has a collinear antiferromagnetic structure with the moments confined in the $c$-plane.

\subsection{Single Crystal Experiment}

The main panel and the inset of Fig. 4(a) show the temperature $(T)$ dependence of the integrated intensity and the typical $\omega$-scan profiles at $\boldsymbol{Q}=(0,1,0)$, respectively. The small intensity at the $\boldsymbol{Q}$-point exists above $T_{N}$, which is originated from the nuclear reflection. With decreasing $T$, a large intensity component due to magnetic ordering appears below $T_{N}$.

Nuclear superlattice reflections are observed at 
(a)

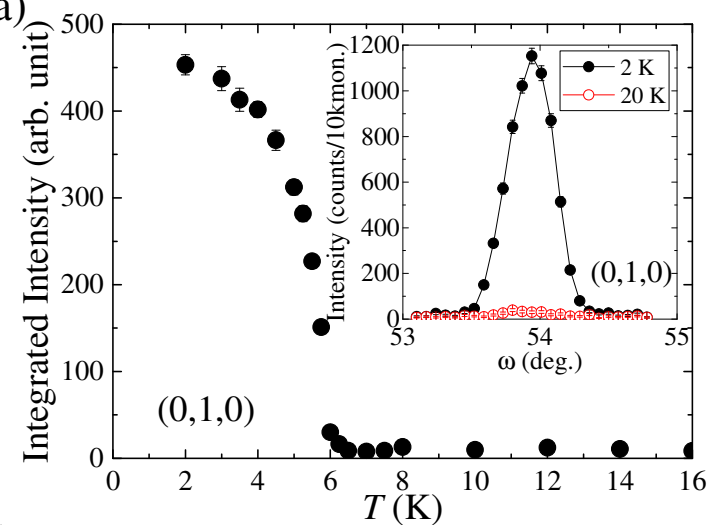

(b)

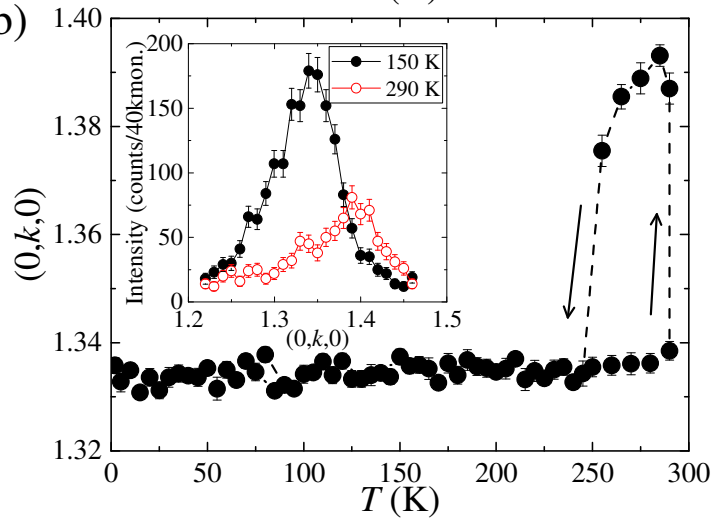

Fig. 4. (color online) (a) $T$-dependence of the integrated intensity at the $\boldsymbol{Q}$-point $(0,1,0)$. Inset shows the typical $\omega$-scan profiles. (b) $T$-dependence of the $k$-value of the superlattice reflection at $(0, k, 0)$. Inset shows the typical profiles along $k$-direction.

$\left(h_{0} \pm \delta, k_{0}, l_{0}\right),\left(h_{0}, k_{0} \pm \delta, l_{0}\right)$, and $\left(h_{0} \pm \delta, k_{0} \pm \delta, l_{0}\right)\left(h_{0}, k_{0}\right.$, and $l_{0}=$ integer number, and $\delta=$ noninteger number). The inset of Fig. 4(b) shows the typical profiles of the superlattice reflection along $k$-direction at $(0, k, 0)$, where $k=1-\delta$. It is clear that the superlattice reflection changes with the temperature. The $T$-dependence of the $k$-value of the superlattice reflection at $(0, k, 0)$ is shown in Fig. 4(b). In the wide $T$ region, the nuclear superlattice reflections with $\delta=1 / 3$ are observed. At around $250 \mathrm{~K}$, the $\delta$-value of the superlattice reflection changes with hysteresis, suggesting that the structural transition takes place at this temperature. ${ }^{33)}$

The observed superlattice peaks are explained by a $3 \times 3 \times 1$ supercell with the space groups $P 2{ }_{1} 2{ }_{1} 2 .{ }^{34)}$ The main purpose of our study is, however, the magnetic structure of $\mathrm{Ca}_{2} \mathrm{CoSi}_{2} \mathrm{O}_{7}$ instead of detailed crystal structure analysis. Since the nuclear intensity is used only for the scaling factor to estimate the magnitude of the magnetic moment in the magnetic structure analysis, the use of the averaged crystal structure is reasonable. Also, the nuclear reflection observed at $(0,1,0)$ is prohibited in $P 2_{1} 2_{1} 2$ and the symmetry of the crystal is further reduced. In the present study, we analyzed the magnetic structure using the averaged space group $P \overline{4} 2{ }_{1} m .^{30)}$

The magnetic diffraction measurements on the $\mathrm{Ca}_{2} \mathrm{CoSi}_{2} \mathrm{O}_{7}$ single crystal were carried out in order to clarify the direction of the magnetic moment in the $c$-plane. At $20 \mathrm{~K}$ and $2 \mathrm{~K}$, the neutron intensities were measured at the $\boldsymbol{Q}$-points equivalent to $(1,2,0)$. The magnetic intensities were obtained by subtracting the integrated intensity at $20 \mathrm{~K}$ from that at $2 \mathrm{~K}$. In order to avoid the neutron absorption effect, the magnetic intensities were divided by the nuclear intensities at each $\boldsymbol{Q}$-point. Then, the magnetic intensities at the equivalent $\boldsymbol{Q}$-points can be compared relatively and accurately on the assumption that the nuclear intensities at the equivalent $\boldsymbol{Q}$-points are equal. The average of the obtained magnetic intensities is normalized to 1.0, and the magnetic intensity at each $\boldsymbol{Q}$-point is plotted as a function of the angle between the scattering vector $\boldsymbol{Q}$ and the $a$-axis in Fig. 5(a). This result shows that the magnetic intensity has the angle dependence in the $c$-plane.

The magnetic neutron scattering is caused by the component of the moment perpendicular to the scattering vector $\boldsymbol{Q}$, and thus the angle dependence of the intensity at equivalent $Q$-points is expressed by the polarization factor $2\left(1-S_{Q}^{2} / S^{2}\right)$. Here the $S_{Q}$ is the component of the magnetic moment parallel to the scattering vector $\boldsymbol{Q}$. Using the formula, the magnetic intensities of the collinear structure with the spins aligned along [100] and [010] directions were calculated; the former is indicated by the blue curve and the latter is by the red curve in Fig. 5(a). In order to explain the angle dependence, we need to consider the imbalance of different magnetic domains. The volume percentages of the domains with the easy axis along [100] and [010] directions are estimated to be 60 and 40\%, respectively, as indicated by the black curve in Fig. 5(a). In contrast, the assumed domains of the magnetic structure with the easy axis along $\langle 110\rangle$ directions, in which the intensity curve shifts by 45 degree from that for [100] and [010] directions, do not reproduce the data. Accordingly, the magnetic structure with the easy axis along $<100>$ directions (Fig. 1(b)) is realized.

The collected 69 magnetic reflections were analyzed by using the above magnetic structure. The intensities were obtained by subtracting the integrated intensity at $20 \mathrm{~K}$ from that at $2 \mathrm{~K}$. We fixed the volume ratio between the domains with the easy axis along [100] and [010] directions as $60: 40$, and we calculated the magnetic cross section $I$. Figure 5(b) shows the observed $I_{\text {obs. }}$ against the model calculation $I_{\text {cal. }}$. The magnitude of the aligned magnetic moment is $3.1( \pm 0.2) \mu_{B}$ which is close to the full moment of $\mathrm{Co}^{3+}$ ion. The observed magnetic intensities are reasonably reproduced by the magnetic structure shown in Fig. 1(b).

Although the canted antiferromagnetic structure is expected from the magnetization curve in Fig. 2, the small ferromagnetic component could not be estimated in our experimental resolution. The residual magnetization for $\boldsymbol{H} \|[100]$ is about $0.02 \mu_{B} / \mathrm{Co}^{3+}$ as shown in the inset of Fig. 2. One of the magnetic domains has the easy axis along [100] and the ferromagnetic component along [010], and another one of the magnetic domains has the easy axis along [010] and the ferromagnetic component along [100]. The actual magnitude of the ferromagnetic component per one Co ion is evaluated to be about $0.04 \mu_{B}$ by considering the magnetic domains. Then, the canted angle from $a$ - or $b$-axis is about 1 degree. As in $\mathrm{Ba}_{2} \mathrm{CoGe}_{2} \mathrm{O}_{7},{ }^{25)}$ this small canting of the neighboring magnetic moments is attributed to the Dzyaloshinskii-Moriya interaction.

\section{Discussion}

We discuss the relationship between the magnetic structure and the multiferroic properties of $\mathrm{Ca}_{2} \mathrm{CoSi}_{2} \mathrm{O}_{7}$. Since the collinear antiferromagnetic structure was identified, the ferro- 
(a)

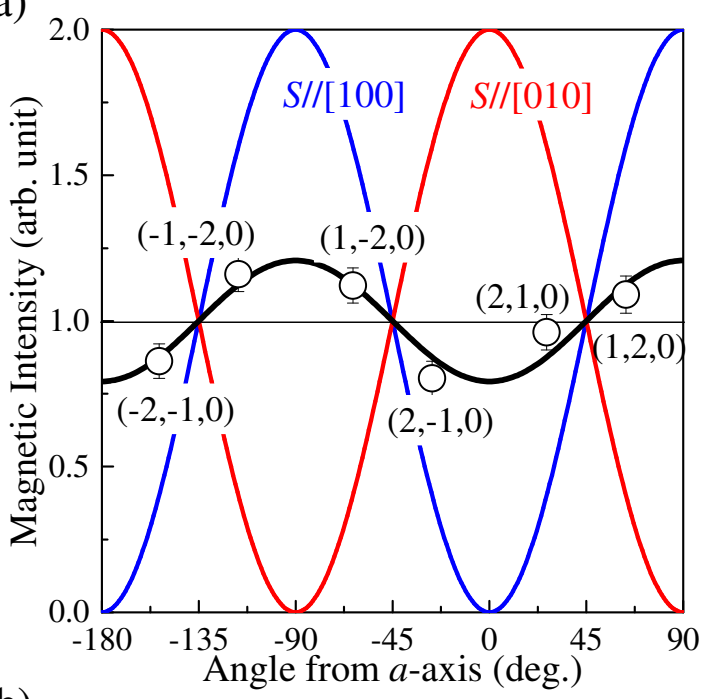

(b)

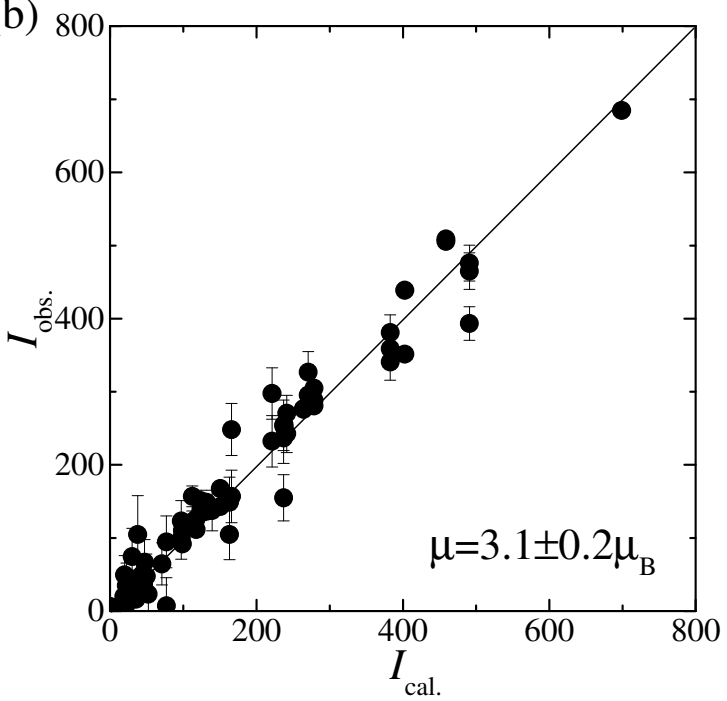

Fig. 5. (color online) (a) Magnetic intensities at the $\boldsymbol{Q}$ points equivalent to $(1,2,0)$ against the angle from the $a$-axis. Calculated intensities for the magnetic structures with the magnetic moments along [100] (red line) and [010] (blue line). Black line shows the angle dependence of the magnetic intensity calculated with the imbalance of the magnetic domains with the easy axis along [100] and [010] directions. (b) Magnetic cross sections obtained by subtracting the integrated intensity at $20 \mathrm{~K}$ from that at $2 \mathrm{~K}$ are shown against those of the model calculation.

electric properties ${ }^{28,29)}$ can be explained neither by the spincurrent model $^{8,12)}$ nor the double exchange mechanism. ${ }^{24,35)}$ Accordingly, we consider the spin-dependent $d$ - $p$ hybridization mechanism ${ }^{11)}$ as in $\mathrm{Ba}_{2} \mathrm{CoGe}_{2} \mathrm{O}_{7} \cdot{ }^{23)}$ In this mechanism, the magnitude and direction of the local electric polarization depend on the direction of the local $\mathrm{Co}^{3+}$ spin and the directions of $\mathrm{Co}-\mathrm{O}$ bondings in $\mathrm{CoO}_{4}$ tetrahedra. This relation is expressed as

$$
\boldsymbol{p} \propto \sum_{i}\left(\boldsymbol{S} \cdot \boldsymbol{e}_{i}\right)^{2} \boldsymbol{e}_{i}
$$

where $\boldsymbol{e}_{i}$ is the vector connecting the Co and the $i$-th $\mathrm{O}$ ions. ${ }^{10,11,23)}$ The electric polarization $\boldsymbol{p}$ points to the $c$-axis from the relationship of Eq. (1) because the Co magnetic moments are confined in the $c$-plane.

In considering Eq. (1) and the crystal structure shown in Fig. 1(a), the dielectric state depends on the direction of the
Co magnetic moment. ${ }^{25,26)}$ The magnetic structure in Fig. 1(b) induces the antiferroelectric structure as shown in Fig. $1(\mathrm{c})$, while the collinear structure with the easy axis along $<110\rangle$ directions induces the ferroelectric state as shown in Fig. 1(d). In $\mathrm{Ca}_{2} \mathrm{CoSi}_{2} \mathrm{O}_{7}$, thus, the antiferroelectric structure must be realized.

Now let us discuss the magnetization data in Fig. 2 based on the collinear structure identified by the present neutron diffraction. Assuming that the dominant in-plane anisotropy in $\mathrm{Ca}_{2} \mathrm{CoSi}_{2} \mathrm{O}_{7}$ is orthorhombic anisotropy and the origin is single-ion type $E\left(\left(S^{[100]}\right)^{2}-\left(S^{[010]}\right)^{2}\right)$ with negative $E$, the spin flop transition is present in the field $\boldsymbol{H} \|[100]$ and it is absent in $\boldsymbol{H} \perp[100]$. In the field $\boldsymbol{H} \|[110]$ which is tilted by $45^{\circ}$ from the easy axis, the spin flop anomaly is strongly suppressed and smeared. This behavior is qualitatively consistent with the the magnetization data exhibiting the anomaly at $H \sim 3.5 \mathrm{~T}$ in $\boldsymbol{H} \|[100]$ and the absence of anomaly in $\boldsymbol{H} \|[110]$, except hysteretic behavior in $|H| \lesssim 1 \mathrm{~T}$. The assumption is, thus, reasonable and the single-ion anisotropy $E$ is the dominant origin for the in-plane anisotropy instead of spin-nematic interaction $J_{p}$ originated from the interaction between local electric polarization.

This contrasts with the isostructural $\mathrm{Ba}_{2} \mathrm{CoGe}_{2} \mathrm{O}_{7}$ which has the same magnetic structure and electric polarization structure. ${ }^{25,26)}$ The origin of the anisotropy in $\mathrm{Ba}_{2} \mathrm{CoGe}_{2} \mathrm{O}_{7}$ is the spin-nematic interaction and the anisotropy has fourfold rotational symmetry. This leads to different type of spin flop; the spins flop from [100] or [010] to [1 $1 \overline{1} 0]$ at $H=0.35$ $\mathrm{T}$ in $\boldsymbol{H} \|[110] .{ }^{25)}$ In fact the symmetry of the $\mathrm{Ba}_{2} \mathrm{CoGe}_{2} \mathrm{O}_{7}$ is also lowered by slight lattice distortion ${ }^{36)}$ and orthorhombic anisotropy in the $c$-plane is allowed. The spin nematic interaction is, however, dominant and exotic type of spin flop transition was exhibited.

While the magnetization in the low- $H$ region was explained by the in-plane anisotropy, distinct anomaly for $\boldsymbol{H} \|[110]$ at 11 $\mathrm{T}$ and non-trivial magnetization plateau for $\boldsymbol{H} \| c$-axis above $18 \mathrm{~T}^{29)}$ cannot be explained. Small energy scale of the singleion anisotropy $D$ is suggested by the magnetization measurements, ${ }^{29,37,38)}$ and proximity of the $D$ and the exchange interaction would be the origin for the anomalous behavior in a high field. Detailed study on spin dynamics will be the key to the understanding of the relationship among the magnetization, the single-ion anisotropy, and the exchange interaction.

\section{Conclusions}

Combination of neutron diffraction experiments on polycrystalline and single-crystal samples has revealed magnetic structure of the multiferroic $\mathrm{Ca}_{2} \mathrm{CoSi}_{2} \mathrm{O}_{7}$. The former experiment has shown the antiferromagnetic collinear structure in the $c$-plane. The latter experiment including precise measurements on several equivalent Bragg peaks has shown that the spin directions are along $\langle 100\rangle$. Antiferroelectric structure has been derived from the discussion on the multiferroic property on the basis of the spin-dependent $d$ - $p$ hybridization mechanism. The origin of the magnetic anisotropy that determines the multiferroic property has been discussed, and the orthorhombic anisotropy $E$ is dominant in the in-plane anisotropy.

Travel expenses to SINQ for the neutron experiments were supported by General User Program for Neutron Scattering Experiments, Institute for Solid State Physics, The University 
of Tokyo (proposal no. 15519). This work was supported in part by KAKENHI (15K05123 and 15K05145) from MEXT, Japan.

1) M. Fiebig, J. Phys. D: Appl. Phys. 38, R123 (2005)

2) W. Eerenstein, N. D. Mathur, and J. F. Scott, Nature 442, 759 (2006).

3) T. Kimura, Annu. Rev. Mater. Res. 37, 387 (2007).

4) S.-W. Cheong and M. Mostovoy, Nature Mater. 6, 13 (2007).

5) T. Goto, T. Kimura, G. Lawes, A. P. Ramirez, and Y. Tokura, Phys. Rev. Lett. 92, 257201 (2004).

6) T. Kimura, G. Lawes, and A. P. Ramirez, Phys. Rev. Lett. 94, 137201 (2005).

7) Y. Kitagawa, Y. Hiraoka, T. Honda, T. Ishikura, H. Nakamura, and T. Kimura, Nature Mater. 9, 797 (2010).

8) H. Katsura, N. Nagaosa and A. V. Balatsky, Phys. Rev. Lett. 95, 057205 (2005).

9) M. Mostovoy, Phys. Rev. Lett. 96, 067601 (2006).

10) C. Jia, S. Onoda, N. Nagaosa, and J. H. Han, Phys. Rev. B. 76, 144424 (2007)

11) T. Arima, J. Phys. Soc. Jpn. 76, 073702 (2007).

12) Y. Yamasaki, H. Sagayama, T. Goto, M. Matsuura, K. Hirota, T. Arima and Y. Tokura, Phys. Rev. Lett. 98, 147204 (2007).

13) I. Cabrera, M. Kenzelmann, G. Lawes, Y. Chen, W. C. Chen, R. Erwin, T. R. Gentile, J. B. Leao, J.W. Lynn, N. Rogado, R. J. Cava, and C. Broholm, Phys. Rev. Lett. 103, 087201 (2009).

14) M. Soda, T. Ishikura, H. Nakamura, Y. Wakabayashi, and T. Kimura, Phys. Rev. Lett. 106, 087201 (2011).

15) M. Soda, K. Kimura, T. Kimura, M. Matsuura, and K. Hirota, J. Phys. Soc. Jpn. 78, 124703 (2009).

16) K. Kimura, H. Nakamura, K. Ohgushi, and T. Kimura, Phys. Rev. B 78, 140401(R) (2008)

17) T. Kimura, T. Goto, H. Shintani, K. Ishizaka, T. Arima, and Y. Tokura, Nature 426, 55 (2003).

18) H. Sagayama, K. Taniguchi, N. Abe, T. Arima, M. Soda, M. Matsuura, and K. Hirota, Phys. Rev. B 77, 220407(R) (2008).

19) M. Soda, K. Kimura, T. Kimura, and K. Hirota, Phys. Rev. B 81, 100406(R) (2010)
20) T. Kurumaji, K. Ohgushi, and Y. Tokura, Phys. Rev. B. 89, 195126 (2014).

21) S. Hayashida, M. Soda, S. Itoh, T. Yokoo, K. Ohgushi, D. Kawana, and T. Masuda, Phys. Proc. 75, 127 (2015).

22) S. Hayashida, M. Soda, S. Itoh, T. Yokoo, K. Ohgushi, D. Kawana, and T. Masuda, J. Phys.: Conf. Ser. 746, 012059 (2016).

23) H. Murakawa, Y. Onose, S. Miyahara, N. Furukawa, and Y. Tokura, Phys. Rev. Lett. 105, 137202 (2010).

24) B. Lorenz, Y. Wang, and C. Chu, Phys. Rev. B 76, 104405 (2007)

25) M. Soda, M. Matsumoto, M. Månsson, S. Ohira-Kawamura, K. Nakajima, R. Shiina, and T. Masuda, Phys. Rev. Lett. 112, 127205 (2014).

26) M. Soda, S. Hayashida, B. Roessli, M. Månsson, J. S. White, M. Matsumoto, R. Shiina, and T. Masuda, Phys. Rev. B 94, 094418 (2016).

27) I. Kézsmárki, N. Kida, H. Murakawa, S. Bordács, Y. Onose, and Y. Tokura, Phys. Rev. Lett. 106, 057403 (2011).

28) M. Akaki, J. Tozawa, D. Akahoshi, and H. Kuwahara, J. Phys.: Conf. Ser. 150, 042001 (2009).

29) M. Akaki, H. Kuwahara, A. Matsuo, K. Kindo, and M. Tokunaga, J. Phys. Soc. Jpn. 83, 093704 (2014)

30) K. Kusaka, K. Hagiya, M. Ohmasa, Y. Okano, M. Mukai, K. Iishi, and N. Haga, Phys. Chem. Minerals 28, 150 (2001).

31) A. Zheludev, T. Sato, T. Masuda, K. Uchinokura, G. Shirane, and B. Roessli, Phys. Rev. B. 68, 024428 (2003).

32) P. J. Brown, International Tables for Crystallography, ed. by A. J. C. Wilson (Kluwer, Dordrecht, 1992) vol. C, chap. 4.

33) K. Kusaka, J. Crystal. Soc. Jpn. 49, 129 (2007).

34) A. Sazonov, V. Hutanu, M. Meven, G. Roth, I. Kézsmárki, H. Murakawa, Y. Tokura, and B. Náfrádi, Acta Cryst. B72, 126 (2016).

35) I. A. Sergienko, C Sen, and E. Dagotto, Phys. Rev. Lett. 97, 227204 (2006).

36) V. Hutanu, A. Sazonov, H. Murakawa, Y. Tokura, B. Náfrádi, and D. Chernyshov, Phys. Rev. B 84, 212101 (2011).

37) H. T. Yi, Y. J. Choi, S. Lee, and S.-W. Cheong, Appl. Phys. Lett. 92, 212904 (2008).

38) K. Penc, J. Romhányi, T. Rõõm, U. Nagel, A. Anta, T. Fehér, A. Jánossy, H. Engelkamp, H. Murakawa, Y. Tokura, D. Szaller, S. Bordács, and I. Kezsmárki, Phys. Rev. Lett. 108, 257203 (2012). 